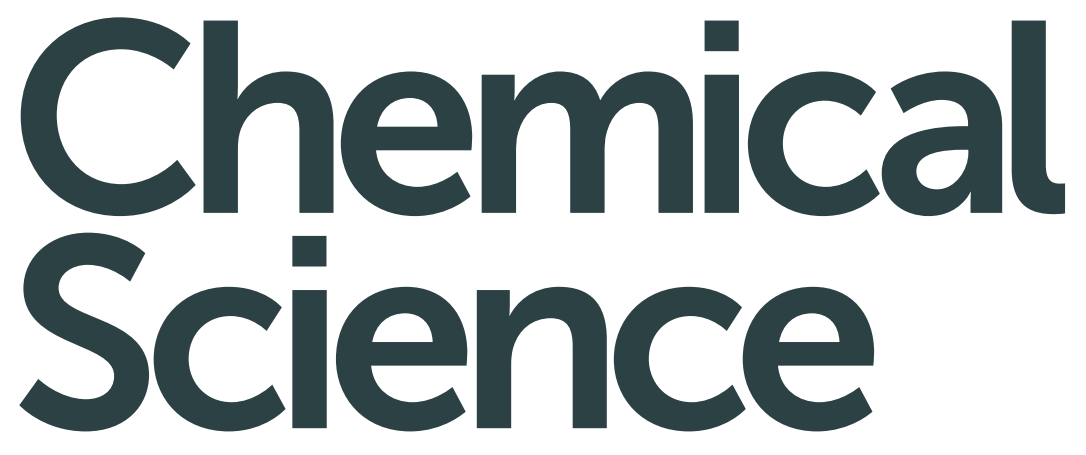

www.rsc.org/chemicalscience
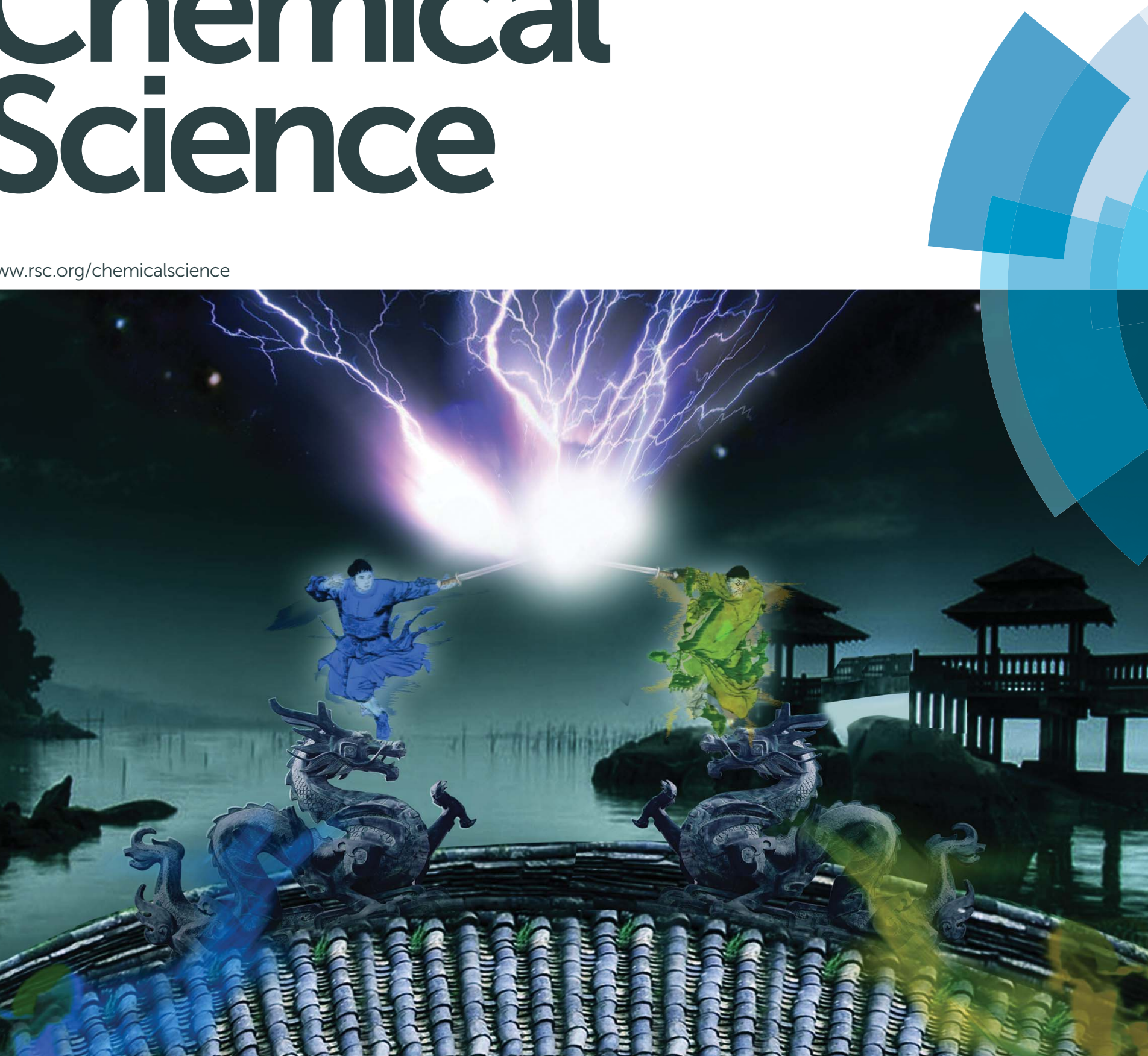

c c c

rock

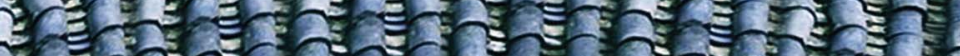




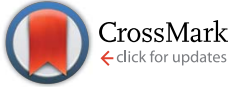

Cite this: Chem. Sci., 2016, 7, 6325

\title{
Foldable glycoprobes capable of fluorogenic crosslinking of biomacromolecules $\uparrow$
}

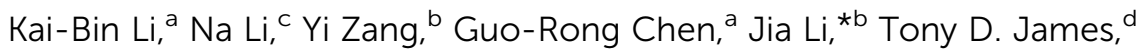 \\ Xiao-Peng $\mathrm{He}^{\star a}$ and $\mathrm{He} \mathrm{Tian}^{\mathrm{a}}$
}

Small-molecular probes capable of monitoring and interfering with the activity of biomacromolecules such as polysaccharides, nucleotides and proteins - are of paramount importance to the advancement of life science. However, such probes that can detect and simultaneously modulate the construction of biomacromolecules are elusive. Here we report a fluorogenic, foldable glycoprobe that can recognize and assemble a protein receptor in a synchronous fashion. The glycoprobe synthesized by introducing a glycoligand (for protein recognition) to a bola-type bis-fluorophore conjugate shows a "self-shielded" fluorescence in the folded state. Association with a receptor protein rapidly unfolds the probe, releasing a fluorophore capable of crosslinking the proteins - as determined using small-angle X-ray scattering thereby producing a unique fluorescent supramolecular construct. We have demonstrated the use of the foldable glycoprobe in order to track the endocytic cycle of a transmembrane receptor.

Received 28th May 2016 Accepted 11th July 2016

DOI: $10.1039 / \mathrm{c} 6 \mathrm{sc} 02366 \mathrm{e}$

www.rsc.org/chemicalscience

progression and metastasis of cancers, ${ }^{16-20}$ thereby highlighting the importance of probing RMEs processes for disease diagnosis and therapy.

Here we report a single-molecular glycoprobe capable of The ability to effectively identify and control the function of natural biomacromolecules, including nucleotides, proteins and polysaccharides, is of paramount importance to the advancement of life science. Indeed, the past few decades have seen the development of a variety of chemical probes to detect biomolecular targets and therapeutic agents to suppress their activities..$^{1-5}$ However, molecular tools that can simultaneously monitor and modulate the activity of biomacromolecules have been elusive.

Receptor proteins are a class of macromolecules closely related to a number of biological processes, for example the initiation of downstream cellular pathways through selective receptor-ligand interactions. ${ }^{6-9}$ They can also transport endogenous and exogenous ligand molecules from the plasma to the intracellular milieu (for example to the lysosome for degradation), ${ }^{10,11}$ maintaining the balance of these species in the blood stream. However, in some cases Receptor Mediated Entries (RMEs) are also implicated in the invasion of viruses ${ }^{12-15}$ and

\footnotetext{
${ }^{a}$ Key Laboratory for Advanced Materials \& Institute of Fine Chemicals, School of Chemistry and Molecular Engineering, East China University of Science and Technology, 130 Meilong Rd., Shanghai 200237, PR China.E-mail:xphe@ecust.edu.cn ${ }^{b}$ National Center for Drug Screening, State Key Laboratory of Drug Research, Shanghai Institute of Materia Medica, Chinese Academy of Sciences, 189 Guo Shoujing Rd., Shanghai 201203, PR China. E-mail: jli@simm.ac.cn

${ }^{c}$ National Center for Protein Science Shanghai, Shanghai Institutes of Biological Sciences, Chinese Academy of Sciences, Shanghai 200031, PR China

${ }^{d}$ Department of Chemistry, University of Bath, Bath, BA2 7AY, UK

$\dagger$ Electronic supplementary information (ESI) available: Experimental section, additional figures and spectral copies. See DOI: 10.1039/c6sc02366e
} Through the coupling of a glycoligand to a bis-fluorophore conjugate we have produced a foldable glycoprobe with minimal fluorescence in an aqueous medium (Fig. 1a). Subsequently, association of the probe with a selective receptor protein rapidly enhances the fluorescence by unfolding the probe (Fig. 1b), and this releases an additional fluorophore "hand" which can facilitate cross-linking of the proteins to form unique supramolecular assemblies, as determined by smallangle X-ray scattering (SAXS) (Fig. 1c). Furthermore, the foldable glycoprobe has proven effective in tracking the endocytic cycle of a transmembrane receptor protein.

\section{Results and discussion}

The folded glycoprobes were synthesized by introducing a glycoligand (galactose that can be recognized by galactose-selective receptor proteins) to a bis-fluorophore conjugate using a click reaction. ${ }^{21,22}$ To synthesize the conjugate, a naphthalimide was grafted to 1-pyrenebutyrate via a flexible alkyl chain to facilitate intramolecular interaction of the two fluorophores (Fig. 1, Scheme S1†) ${ }^{23-25}$ Therefore, we prepared LKB1, LKB2, LKB3 and LKB4 with increasing linker chain length between the glycoligand and the conjugate, and LKB5 using a mannose instead of galactose (Fig. 1 and Scheme S2 $\dagger$ ).

With the glycoprobes in hand, we measured their fluorescence in the absence and presence of a galactose-selective 
a

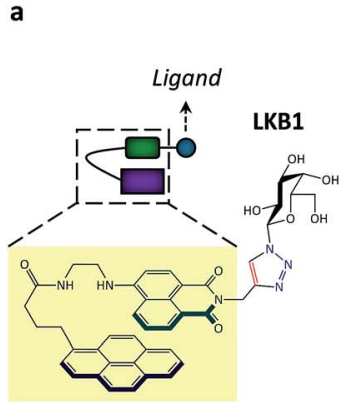

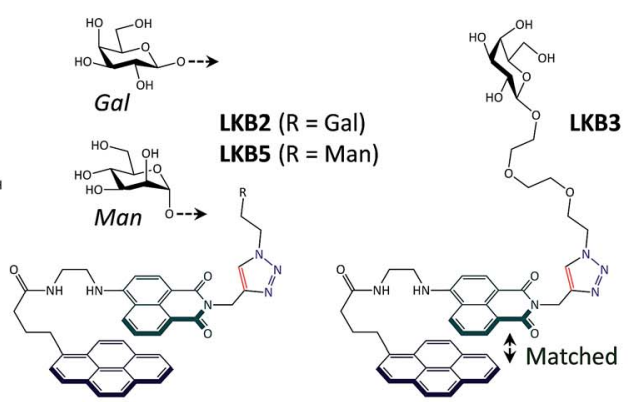

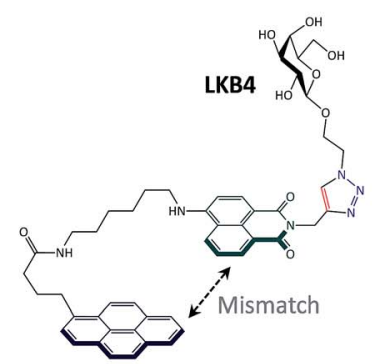

b

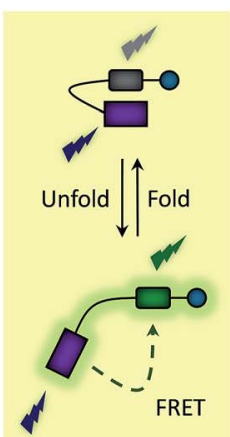

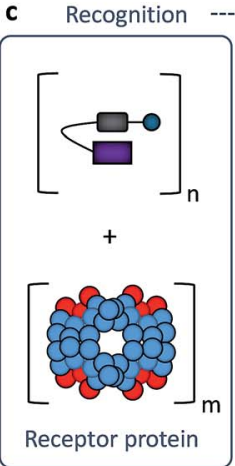

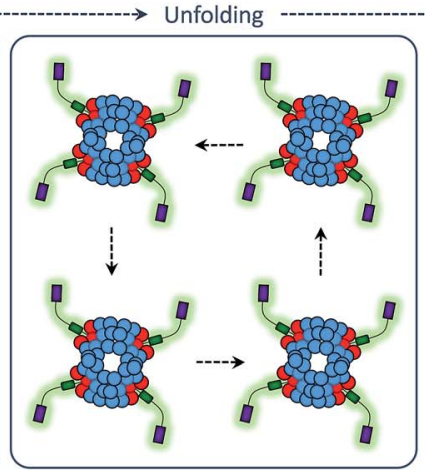

Supramolecular "hand-in-hand"

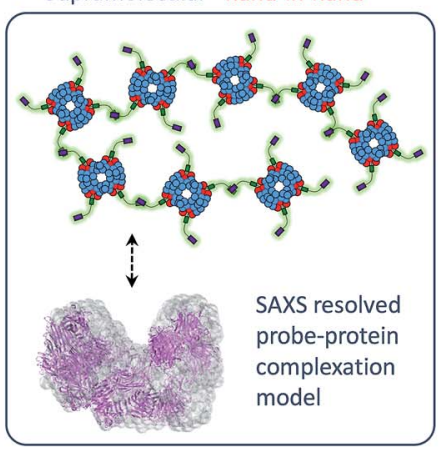

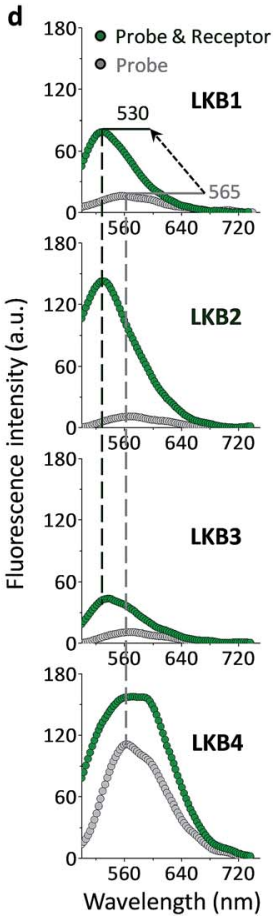

Fig. 1 Structures of the foldable glycoprobes synthesized and schematic illustration of their ability to crosslink receptor proteins. (a) Structure of the folded glycoprobes LKB1-LKB5. Schematic illustration of (b) the folded and unfolded states of glycoprobe and (c) supramolecular assembly of the glycoprobe with a protein receptor. (d) Fluorescence spectra of LKBs (5 $\mu \mathrm{M}$ in $0.05 \mathrm{M} \mathrm{PBS,} \mathrm{pH} \mathrm{7.4;} \mathrm{excitation} \mathrm{wavelength:} 345 \mathrm{~nm}$ ) in the absence and presence of a receptor protein, peanut agglutinin (PNA, $25 \mu \mathrm{M}$ ).

lectin, peanut agglutinin (PNA), in an aqueous buffer solution (Tris-HCl, pH 7.4). We determined that LKB1, LKB2 and LKB3 displayed minimal fluorescence in the buffer medium with excitation at $345 \mathrm{~nm}$, whereas LKB4 had strong fluorescence with an emission maxima at $c a .565 \mathrm{~nm}$ (Fig. 1d). Addition of PNA caused a large fluorescence enhancement of LKB1-LKB3 with a blue-shifted emission maxima ( $c a .530 \mathrm{~nm}$ ), but only slight fluorescence enhancement with LKB4 (Fig. 1d). Among the three probes with weak initial fluorescence, LKB2 displayed the largest fluorescence enhancement in the presence of PNA. This suggests that the association of LKB2 with PNA is optimal. In contrast, an increase of linker chain length between the two fluorophores led to an increased initial fluorescence of LKB4, probably suggesting sub-optimal matching between the naphthalimide and pyrene.

LKB2 with the best PNA sensitivity was selected to investigate the mechanism by which the fluorescence of the probe is enhanced (Fig. S1 $\dagger$ ). We first determined that the absorbance (A) band of LKB2 red-shifted with respect to the unconjugated naphthalimide (N) and pyrene (P) (Scheme S1†) (Fig. S1a and Scheme S1 $\dagger$ ). This suggests an intimacy between the two fluorophores. ${ }^{26,27}$ Shown in Fig. S1b $\dagger$ are the stacked absorbance (A) and emission (E) spectra of $\mathbf{P}$ and $\mathbf{N}$, implying a possibility of Förster resonance energy transfer (FRET) from $\mathbf{P}$ to $\mathbf{N}$ because of good overlap between the emission band of the former with the absorbance band of the latter. Using these observations we deduce that the two hydrophobic fluorophores are stacked in aqueous solution to adopt an excimer-like, folded conformation with red-shifted (with respect to naphthalimide) and weak fluorescence emission. ${ }^{28,29}$ Subsequent unfolding of the probe through association with a protein produces a typical naphthalimide emission ${ }^{30}$ through FRET, upon excitation of pyrene (345 nm) (Fig. 1b). This deduction was corroborated by two additional assays. Firstly, we determined that an increase of temperature $(T)$ caused the emission peak of the probe to gradually blue-shift (Fig. S1c†). A similar blue-shift was observed for the probe in a range of organic solvents (Org) when compared to that in full aqueous buffer (Aq) (Fig. S1d †). Both assays imply that the folded structure of the probe could be extended and opened by tuning of the solvent conditions.

Next, we determined that the fluorescence enhancement of LKB2 and the mannose-appended LKB5 was dependent on the concentration of PNA (Fig. S1e $\dagger$ ) and a mannose-selective lectin, concanavalin A (Con A) (Fig. S1f $\dagger$ ), respectively. In addition, the fluorescence enhancement was observed to be specific for selective lectin receptors (LKB2 for PNA and LKB5 for Con A and another mannose-selective lectin, lens culimaris agglutinin [LCA]) over a panel of unselective lectins and proteins including wheat germ agglutinin, pisum sativum lectin, bovine serum albumin, pepsin, lysin, human cytochrome $\mathrm{c}$ and ribonuclease A (Fig. S1g and $h \dagger$ ).

With the biospecificity of the glycoprobes determined, we sought to determine the mode of association between the probe and PNA using SAXS, a technique that can provide detailed structural information for macromolecules. ${ }^{31,32}$ On the basis of the single merged X-ray scattering profile obtained from PNA solutions with or without LKB2, ab initio low-resolution models were calculated (Fig. 2c). The results suggest a gradual 
a

Small-angle $X$-ray scattering (SAXS) resolved, solution based ligand-receptor complexes

.

aggregation of PNA in the presence of increasing concentrations of the glycoprobe, forming an octamer with a final probe/ protein ratio of $1 / 15(\mathrm{w} / \mathrm{w})$ (Fig. 2a). Fig. 2b shows the interatomic distance distribution function, $P(r)$, which similarly indicates an increase in maximum diameter $\left(D_{\max }\right)$ of the protein with increasing probe concentration. Recently, a solidstate complex structure formed between a glucosyl rhodamine and Con $\mathrm{A}$ has been resolved by X-ray crystallography, ${ }^{33}$ revealing that while the glucosyl moiety docks into the carbohydrate recognition domain of Con $\mathrm{A}$, the rhodamine tail can dimerize and facilitate aggregation of the proteins. With this $\mathrm{X}$ ray crystal data and our SAXS results we suggest that association of the glycoprobe with PNA can unfold the bis-fluorophore conjugate, releasing the pyrene tail. These released pyrene tails can then stack with each other to crosslink the proteins, forming protein aggregates as determined using SAXS (Fig. 1c).

Having established the solution-based glycoprobe-protein complexation, we investigated the interaction of LKB2 with a transmembrane receptor at the cellular level. We used a human liver cancer cell line that expresses a galactose-selective asialoglycoprotein receptor (ASGPr) ${ }^{34-36}$ and other cell lines including HeLa (human cervix cancer), A549 (human lung cancer) and MGC803 (human gastric cancer) as controls. We observed that incubation of $\mathbf{L K B 2}$ with the cells resulted in a strong fluorescence for Hep-G2, but not in the control cells (Fig. 3a and b). This is in agreement with the ASGPr expression level of the cell lines determined by real-time quantitative polymerase chain reaction (Fig. 3c). Meanwhile, treatment of the cells with LKB5 bearing a mannose precursor did not produce fluorescence in all the cell lines used (Fig. 3a and b).

We also used a modified Hep-G2 cell line (sh-ASGPr) with a suppressed ASGPr expression by sh-RNA plasmid transfection $^{37}$ with LKB2. The result suggested that knockdown of ASPGr significantly reduces the fluorescence imaging effect of the glycoprobe (Fig. 3d and f), and the fluorescence intensity produced by LKB2 in sh-ASGPr and control (Hep-G2 transfected with a scrambled sh-RNA) is consistent with the receptor expression level of the two cell lines (Fig. 3g). A competition assay by pre-treatment of free lactose with Hep-G2 showed that the fluorescence of LKB2 in Hep-G2 could be gradually reduced with increasing lactose concentrations (Fig. 3e and h). Meanwhile, a cell viability assay suggested that both LKB2 and LKB5 were not toxic to Hep-G2 and sh-ASGPr at a concentration fivefold higher than that used for cell imaging (Fig. S2 $\dagger$ ). These data suggest that the glycoprobe can be used to target a transmembrane receptor for cell imaging experiments. 

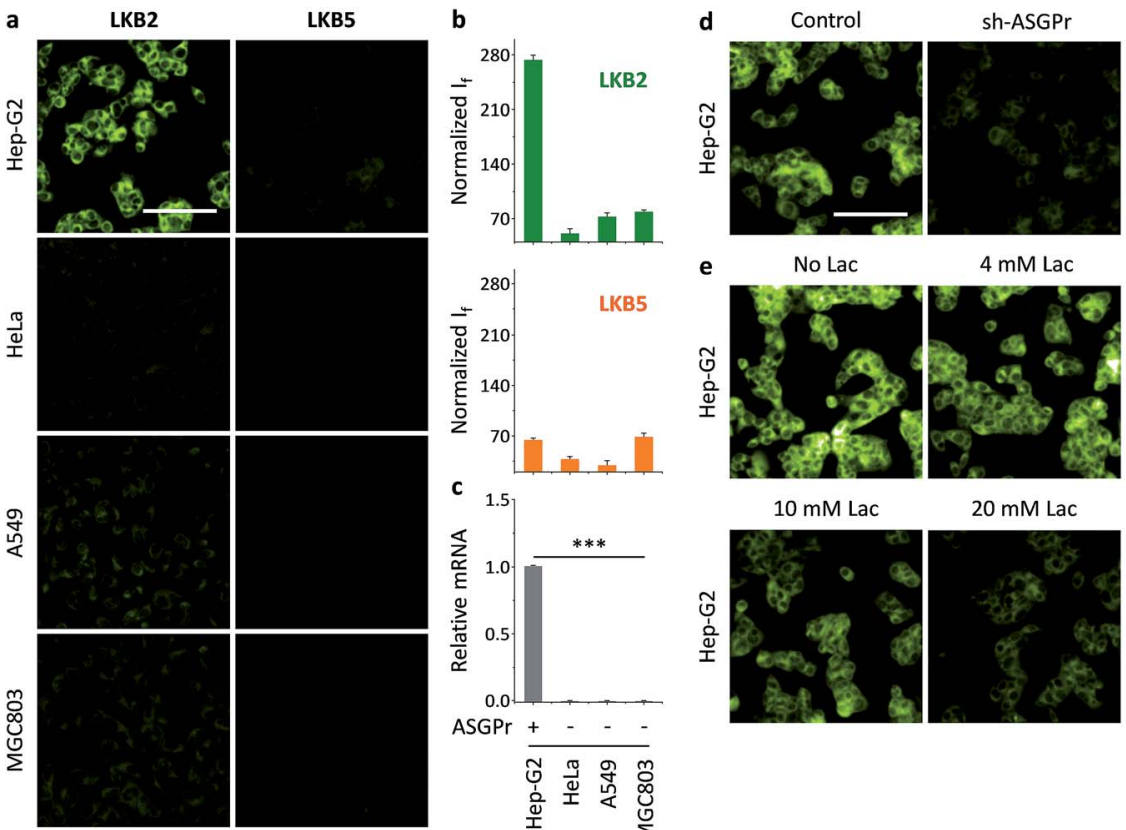

$4 \mathrm{mM} \mathrm{Lac}$
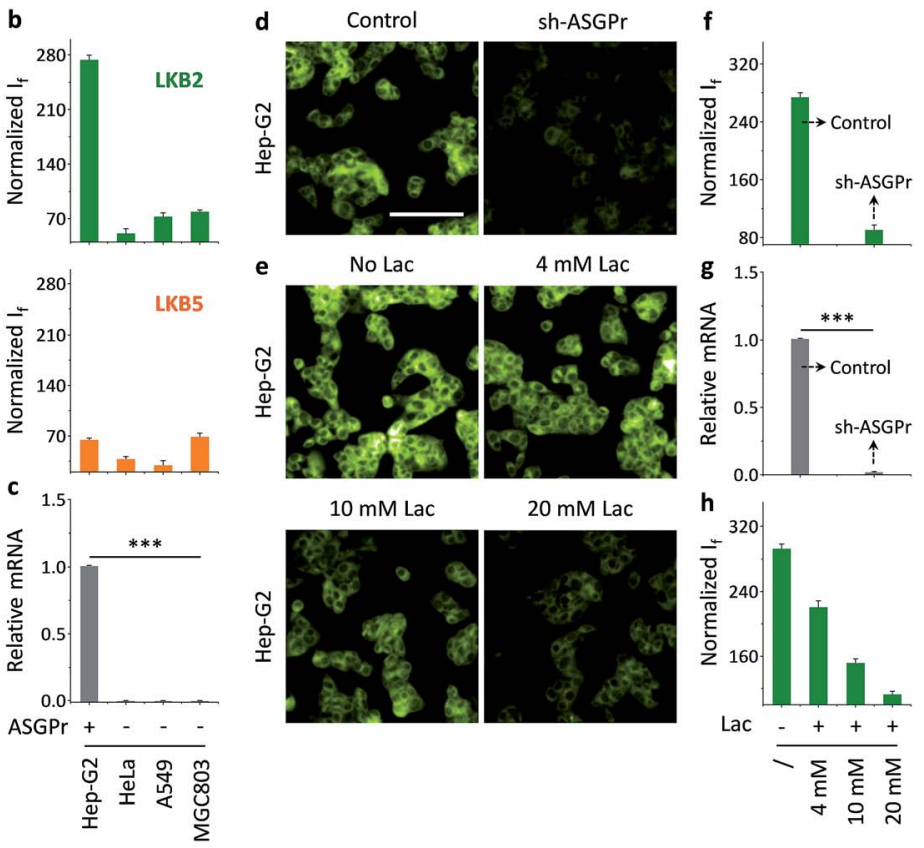

Fig. 3 Receptor-targeting cell imaging of foldable glycoprobes. Fluorescence imaging (a) and quantification (b) of LKB2 (20 $\mu$ M) and LKB5 (20 $\mu \mathrm{M}$ ) for different human cancer cell lines (Hep-G2 = human liver cancer; HeLa = human cervical cancer; A549 = human lung cancer; MGC803 = human gastric cancer). (c) Relative mRNA level of different cancer cells determined by real-time quantitative polymerase chain reaction (RTqPCR) $(* * * P<0.001)$. Fluorescence imaging (d) and quantification (f) of LKB2 $(20 \mu \mathrm{M})$ for Hep-G2 with (sh-ASGPr) or without (control) knockdown of ASGPr (asialoglycoprotein receptor). (g) Relative mRNA level of sh-ASGPr and control determined by RT-qPCR (*** $P<0.001$ ). Fluorescence imaging (e) and quantification (h) of LKB2 $(20 \mu \mathrm{M})$ for Hep-G2 cells preincubated with increasing lactose (Lac). For all fluorescence images, the excitation wavelength was $345 \mathrm{~nm}$ and emission channel 450-550 nm (scale bar: $100 \mu \mathrm{m}$, which is applicable to all images).
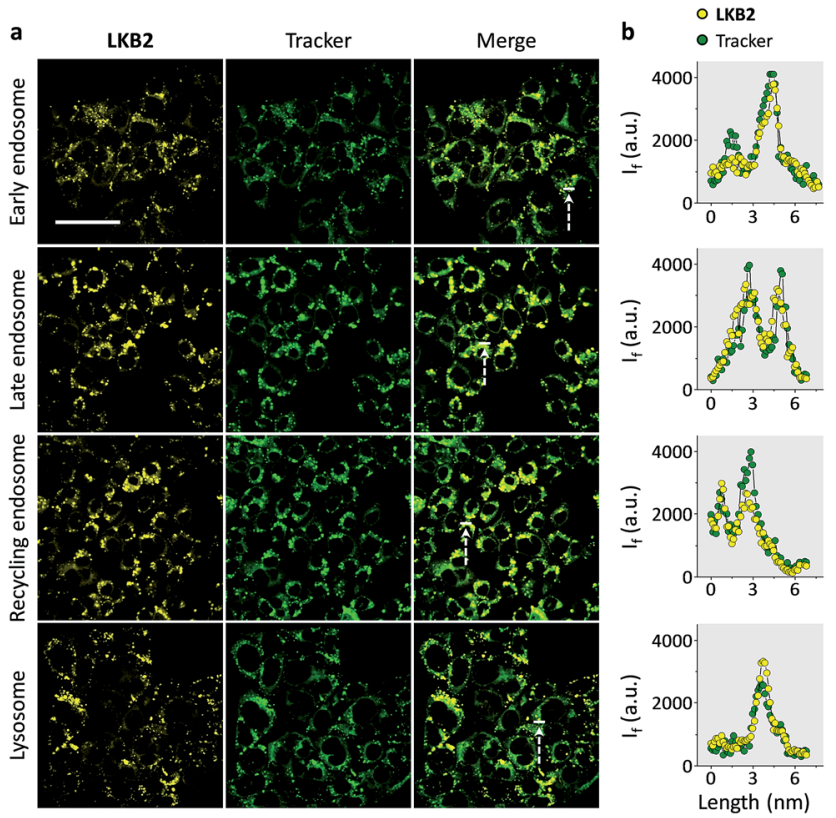

Fig. 4 Tracking the endocytic cycle of a transmembrane receptor by foldable glycoprobes. Fluorescence imaging (a) and quantitative colocalization (b) (the line-cross sections shown in the merged images were used for quantification) of LKB2 $(80 \mu \mathrm{M})$ and green fluorescence protein (GFP)-tagged antibody trackers of different subcellular compartments using confocal scanning laser microscopy (excitation: $400 \mathrm{~nm}$; emission channel: $450-550 \mathrm{~nm}$; scale bar: $40 \mu \mathrm{m}$, which is applicable to all images).
Finally, we tested the ability of LKB2 to track the endocytosis of ASGPr by confocal laser scanning microscopy. Typical endocytic pathways for ASGPr have been suggested, which depend on the formation of early endosomes after ligandreceptor binding and a segregation of ligand and receptor, where the ligand is delivered by late endosomes to the lysosome for degradation and receptor recycled to cell surface by recycling endosomes. ${ }^{10}$ As a result, we used Hep-G2 cells transfected with different GFP (green fluorescence protein)-tagged antibody trackers of the subcellular compartments to monitor the glycoprobe internalization. We observed strong fluorescence signals in all the compartments tracked (Fig. 4a); the fluorescence of LKB2 co-localized well with those of GFP trackers (Fig. 4b). We also used a control naphthalimide galactoprobe (LKB6) without the pyrene tail for cell imaging (Fig. S3†). The results indicated that the control compound had a much weaker Hep-G2 imaging effect than LKB2, probably suggesting the importance of the foldable motif of the latter to underpin the receptor binding. Although more evidence is needed to elucidate the dynamic and complex receptor-probe binding processes in cells, the confocal imaging results obtained here highlight the effectiveness of the foldable probe to track the intracellular localizations of receptor proteins.

\section{Conclusions}

With this research we have developed a foldable glycoprobe that can be used to simultaneously detect and modulate the 
construction of protein receptors. While the probe displayed specific fluorescence enhancements in the presence of a selective receptor in aqueous buffer solution, results obtained from SAXS suggest a concentration-dependent crosslinking of proteins by the probe. The unique probe-protein oligomeric complexation determined represents an exceptional example of protein aggregation by small-molecular probes. The observation that the glycoprobe has proven amenable to tracking the endocytic cycle of a transmembrane receptor highlights its potential for use in elucidating the intracellular localization of functional receptor proteins. This research also offers a new insight into the construction of supramolecular constructs based on the bio-recognition between a foldable small-molecular probe and biomacromolecules.

\section{Acknowledgements}

This research is supported by the 973 project (2013CB733700), the National Natural Science Foundation of China (21572058, 21576088 and 31200617), the Science and Technology Commission of Shanghai Municipality (15540723800) and the Shanghai Rising-Star Program (16QA1401400). The Catalysis And Sensing for our Environment (CASE) network is thanked for research exchange opportunities. T. D. J. thanks ECUST for a guest professorship. We also thank the staff from BL17B/ BL18U1/BL19U1/BL19U2/BL01B beamline of National Center for Protein Sciences Shanghai (NCPSS) at Shanghai Synchrotron Radiation Facility, for assistance during data collection.

\section{Notes and references}

1 For a recent themed issue, see: C. J. Chang, T. Gunnlaugsson and T. D. James, Chem. Soc. Rev., 2015, 44, 4176-4178.

2 For a recent themed issue, see: C. J. Chang, T. Gunnlaugsson and T. D. James, Chem. Soc. Rev., 2015, 44, 4484-4486.

3 R. Zou, Q. Wang, J. Wu, J. Wu, C. Schmuck and H. Tian, Chem. Soc. Rev., 2015, 44, 5200-5219.

4 H. N. Kim, Z. Guo, W. Zhu, J. Yoon and H. Tian, Chem. Soc. Rev., 2011, 40, 79-93.

5 X.-P. He, Y. Zang, T. D. James, J. Li and G.-R. Chen, Chem. Soc. Rev., 2015, 44, 4239-4248.

6 D. M. Rosenbaum, S. G. F. Rasmussen and B. K. Kobika, Nature, 2009, 459, 356-363.

7 T. B. H. Geijtenbeek and S. I. Gringhuis, Nat. Rev. Immunol., 2009, 9, 465-479.

8 L. Rizzetto, M. Kuka, C. De Fillippo, A. Cambi, N. G. Netea, L. Beltrame, G. Napolitani, M. G. Torcia, U. D'Oro and D. Cavalieri, J. Immunol., 2010, 184, 4258-4268.

9 T. Lawrence and G. Natoli, Nat. Rev. Immunol., 2011, 11, 750761.

10 P. H. Weigel and J. H. N. Yik, Biochim. Biophys. Acta, 2002, 1572, 341-363.

11 D. C. Kilpatrick, Biochim. Biophys. Acta, 2002, 1572, 187-197.

12 A. Dotzauer, U. Gebhardt, K. Bieback, U. Göttke, A. Kracke, J. Mages, S. M. Lemon and A. Vallbracht, J. Virol., 2000, 74, 10950-10957.
13 G. R. Vasta, Nat. Rev. Microbiol., 2009, 7, 424-438.

14 M. Mohamadzadeh, L. Chen and A. L. Schmaljohn, Nat. Rev. Immunol., 2007, 7, 556-567.

15 J. Prieto, I. Melero and B. Sangro, Nat. Rev. Gastroenterol. Hepatol., 2015, 12, 681-700.

16 F.-T. Liu and V. A. Rabinovich, Nat. Rev. Cancer, 2005, 5, 2941.

17 S. Nakahara and A. Raz, Adv. Anticancer Agents Med. Chem., 2008, 8, 22-36.

18 S. S. Pinho and C. A. Reis, Nat. Rev. Cancer, 2015, 15, 540555.

19 K. M. Mahoney, P. D. Rennert and G. J. Freeman, Nat. Rev. Drug Discovery, 2015, 14, 561-584.

20 K. Palucka and J. Banchereau, Nat. Rev. Cancer, 2015, 12, 265-277.

21 V. V. Rostovtsev, L. G. Green, V. V. Fokin and K. B. Sharpless, Angew. Chem., Int. Ed., 2002, 42, 2596-2599.

22 C. W. Tornøe, C. Christensen and M. Meldal, J. Org. Chem., 2002, 67, 3057-3064.

23 J. Wu, Y. Zou, C. Li, W. Sicking, I. Piantanida, T. Yi and C. Schmuck, J. Am. Chem. Soc., 2002, 134, 1958-1961.

24 H. Zhang, J. Fan, J. Wang, S. Zhang, B. Dou and X. Peng, J. Am. Chem. Soc., 2013, 135, 11663-11669.

25 X. Wu, X. Sun, Z. Guo, J. Tang, Y. Shen, T. D. James, H. Tian and W. Zhu, J. Am. Chem. Soc., 2014, 136, 3579-3588.

26 R. J. Lindquist, K. M. Kefler, K. E. Brown, S. M. Dyar, E. A. Margulies, R. M. Young and M. R. Wasielewski, J. Am. Chem. Soc., 2014, 136, 14912-14923.

27 M. Son, K. H. Park, C. Shao, F. Würthner and D. Kim, J. Phys. Chem. Lett., 2014, 5, 3601-3607.

28 D. W. Cho, M. Fujitsuka, K. H. Choi, M. J. Park, U. C. Yoon and T. Majima, J. Phys. Chem. B, 2006, 110, 4576-4582.

29 R. Ferreira, C. Baleizão, J. M. Muñoz-Molina, M. N. BerberanSantos and U. Pischel, J. Phys. Chem. A, 2011, 115, 10921099.

30 S. Banerjee, E. B. Veale, C. M. Phelan, S. A. Murphy, G. M. Tocci, L. J. Gillespie, D. O. Frimannsson, J. M. Kelly and T. Gunnlaugsson, Chem. Soc. Rev., 2013, 42, 1601-1618.

31 H. D. T. Mertens and D. I. Svergun, J. Struct. Biol., 2010, 172, 128-141.

32 D. I. Svergun, Biol. Chem., 2010, 391, 737-743.

33 F. Sakai, G. Yang, M. S. Weiss, Y. Liu, G. Chen and M. Jiang, Nat. Commun., 2014, 5, 4634.

34 H.-L. Zhang, X.-L. Wei, Y. Zang, J.-Y. Cao, S. Liu, X.-P. He, Q. Chen, Y.-T. Long, J. Li, G.-R. Chen and K. Chen, Adv. Mater., 2013, 25, 4097-4101.

35 X.-P. He, B.-W. Zhu, Y. Zang, J. Li, G.-R. Chen, H. Tian and Y.-T. Long, Chem. Sci., 2015, 6, 1996-2001.

36 X.-L. Hu, Y. Zang, J. Li, G.-R. Chen, T. D. James, X.-P. He and H. Tian, Chem. Sci., 2016, 7, 4004-4008.

37 J. Yang, X. C. Bo, X. R. Ding, J. M. Dai, M. L. Zhang, X. H. Wang and S. Q. Wang, J. Viral Hepatitis, 2006, 13, 158-165. 